\title{
Blends of Poly (L-Lactic Acid) and Glucose Prepared from Mixture Solution
}

\author{
Dan CAO \\ College of Chemical Engineering \\ Nanjing Forestry University \\ Nanjing, China, 210037 \\ e-mail: 1020233375@qq.com \\ Yinping ZHAO \\ College of Chemical Engineering \\ Nanjing Forestry University \\ Nanjing, China, 210037 \\ e-mail: $869484261 @ q q . c o m$
}

\author{
Qinwei GAO* \\ College of Chemical Engineering \\ Nanjing Forestry University \\ Nanjing, China, 210037) \\ e-mail: gqw@njfu.edu.cn
}

\author{
Wei MING \\ College of Chemical Engineering \\ Nanjing Forestry University \\ Nanjing, China, 210037 \\ e-mail: 1208495019@qq.com
}

\begin{abstract}
Poly (L-lactic acid) (PLLA) and glucose (Glu) were respectively dissolved in $\mathrm{N}, \mathrm{N}$-dimethyl acetamide to prepare solutions. The blends of PLLA and glucose with various ratios were prepared by mixed solution method, and characterized with infrared Fourier transform spectrometry, differential scanning calorimetry, thermal gravity analysis, X-ray diffraction and polarized optical microscopy. The structure, thermal properties and crystallinity of PLLA, glucose and the blends were investigated. The results show that PLLA and glucose in blends are liable to form hydrogen-bonding, and PLLA and glucose are partially compatible. The crystallization of the blends increased with increasing glucose. The addition of glucose can promote thermal degradation of PLLA due to the defect of PLLA crystal caused by glucose impurity or trans-esterification between PLLA and glucose.
\end{abstract}

Keywords-poly(L-lactic acid); glucose ;blend; compatibility

\section{INTRODUCTION}

The biodegradable polymer poly (lactic acid) (PLA) is a kind of important eco-friendly synthetic aliphatic polyesters. Moreover, PLA is non-toxic and non- stimulation polyester. PLA possesses a number of interesting properties, including good mechanical properties biocompatibility, and biodegradability. So PLA can be widely used in biomedical materials, packaging textile, and other applications, due to its good properties and processability ${ }^{[1]}$. However, PLA is too stiff and brittle for its applications at room temperature for its glass transition temperature ranging from 55 to $65^{\circ} \mathrm{C}$. Moreover, because of its poor hydrophilicity and cell affinity, PLA is often modified by copolymerization or blending with other component to improve the properties

The introduction of natural glycosyl compounds into PLA chain can improve poor hydrophilicity, reduce glass transition temperature, but also improve biological recognition performance and biocompatibility of modified PLA materials ${ }^{[2]}$. Glucose with five active hydroxyl groups is non-toxic and safe, strong hydrophilic. Ding ${ }^{[3]}$ applied cellulose fiber to reinforce poly(lactic acid) using injection molding, and studied the crystallization and foaming behaviors of PLA/cellulosic fiber composite foams. Chaiwutthinanl ${ }^{[4]}$ prepared biodegradable plastics from PLA, poly(butylene succinate) and microcrystalline cellulose extracted from waste- cotton fabric with a chain extender, which improved the brittleness and thermal stability of PLA. Gao ${ }^{[5]}$ prepared the blends of poly(L-lactic acid) (PLLA) and ethylcellulose(EC) through chloroform solutions at different ratios. The melting point of PLLA-EC blend decreased slightly, the crystallinity and crystal perfection of PLLA-EC blend decreased notably with increasing EC. The research on PLLA modified by saccharide such as glucose is not sufficient. This paper focused on PLA-Glu blend prepared by mixed solution method. The properties and structure of PLLA-Glu blends were discussed.

\section{EXPERIMENTAL METHOD}

\section{A. Materials}

Poly (L-lactic acid) (PLLA) was purchased from Ningbo Universal Biological Material Co. Ltd. Its viscosity-average molecular weight was $2.5 \times 10^{5} \mathrm{~g} / \mathrm{mol}$. Anhydrous glucose was obtained from Chinese Medicine Group Chemical Reagent Co. Ltd. Analytical methanol and chloroform were both from Shanghai Ling Feng Chemical Reagent Co. Ltd. N,N-Dimethyl acetamide (DMAc) was from Nanjing Chemical Reagent Co., Ltd.

\section{B. Preparation of PLLA-Glu Blends}

PLLA contained various additives in industrial production, such as plasticizer, lubricant and toughening agent, etc. So PLLA was purified first. PLLA was dissolved in chloroform to prepare the solution with mass fraction of 5 $\%$ at room temperature. The filtrate of PLLA solution was precipitated in methanol. Then PLLA precipitate was filtered and dried in the vacuum oven at $50^{\circ} \mathrm{C}$ for $10 \mathrm{~h}$. The obtained PLLA was kept in a desiccator.

PLLA and glucose were respectively dissolved in DMAc at $100^{\circ} \mathrm{C}$ for $1 \mathrm{~h}$ to obtain solutions with $15 \mathrm{wt} \%$ 
concentration. The PLLA-Glu mixture solutions with different PLLA/Glu ratios were obtained by mixing PLLA solution and Glu solution proportionally.

PLLA-Glu mixture solution was stirred and dried at $50^{\circ} \mathrm{C}$ under vacuum until the solvent DMAc was completely drained. PLLA-Glu blend was then obtained.

\section{Characterization of PLLA, Glu and Their Lends}

Fourier transform infrared (FT-IR) spectra were recorded on Spectrum Two Spectrometer (PerkinElmer, USA) with a wavenumber range of $4000-400 \mathrm{~cm}^{-1}$ at a resolution of $4 \mathrm{~cm}^{-1}$. Thermo-gravimetric analysis (TG) was carried out by TA Instruments model Q5000 TGA. The samples were heated from 20 to $600^{\circ} \mathrm{C}$ at a heating rate of $10^{\circ} \mathrm{C} / \mathrm{min}$ under $\mathrm{N}_{2}$ atmosphere. Differential scanning calorimetry (DSC) was performed with DSC-200F3 (NETZSCH Company). The sample was heated from 20 to $250^{\circ} \mathrm{C}$ at a rate of $10^{\circ} \mathrm{C} / \mathrm{min}$ under nitrogen atmosphere at gas flow rate of $60 \mathrm{~mL} / \mathrm{min}$. X-ray diffraction analysis (XRD) was used to observe the crystal structure of obtained PLLA-Glu blends with Rigaku D-MAX/Rb X-ray diffractometer at $40 \mathrm{kV}, 30 \mathrm{~mA}$ with $\mathrm{Cu} \mathrm{K \alpha}$, from $5^{\circ}$ to $50^{\circ}$ by the step of $0.05^{\circ}$ at scanning speed of $5^{\circ} / \mathrm{min}$.

\section{RESUlTS AND DISCUSSION}

\section{A. FT-IR Spectra Analysis of PLLA, Glucose and PLLA-Glu Blends}

FT-IR analysis was carried out to evaluate the interaction between PLLA and Glucose. Fig. 1 displays the FT-IR spectra of PLLA, Glu, and PLLA-Glu blends. The FT-IR spectrum of PLLA shows a peak at $3440 \mathrm{~cm}^{-1}$ due to the stretch vibration of hydrogen-bonded and/or free $\mathrm{OH}$, peaks at $1200-1000 \mathrm{~cm}^{-1}$ to the $\mathrm{C}-\mathrm{O}$ stretch, and peaks at $2850-2960 \mathrm{~cm}^{-1}$ to the stretch of $\mathrm{CH}$ and $\mathrm{CH}_{3}$ groups, and the band at $1758 \mathrm{~cm}^{-1}$ due to $\mathrm{C}=\mathrm{O}$ stretch vibration ${ }^{[6-8]}$. The peaks at 871 and $756 \mathrm{~cm}^{-1}$ respectively represent the amorphous and crystalline phases of PLLA. In the spectrum of glucose (curve7), the strong and broad absorption band at 3410 - $3309 \mathrm{~cm}^{-1}$ is caused by $\mathrm{OH}$ stretch vibration in glucose. The weak peak at $1635 \mathrm{~cm}^{-1}$ is due to hemiacetal $\mathrm{CHO}$ group in glucose, while the peak at $915 \mathrm{~cm}^{-1}$ due to glucose ring ${ }^{[4-5]}$.

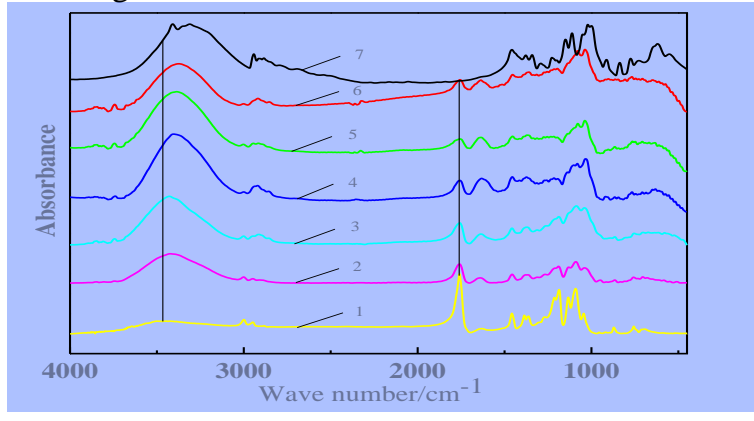

Figure 1. Infrared spectra of PLLA, Glu anfd PLLA-Glu blends Mass ratios of PLLA/Glu: 1. PLLA, 2. 9:1, 3. 8:2, 4. 7:3, 5. 6:4, 6. 5:5, 7. Glu

The FT-IR spectra of PLLA-Glu blends with different proportions all possess characteristic absorption peaks of
Glu and PLLA, but the wavenumber and intensity of these peaks vary with PLLA/Glu ratios of blends, which show that PLLA and Glu can be partly compatible. With increasing Glu, the peak at $1758 \mathrm{~cm}^{-1}$ due to $\mathrm{C}=\mathrm{O}$ group shifts to lower wavenumber, which may be caused by the interaction between $\mathrm{C}=\mathrm{O}$ group of PLLA and $\mathrm{OH}$ group of Glu through hydrogen bonding. With increasing Glu, the intensity of the band at $3300 \sim 3500 \mathrm{~cm}^{-1}$ due to $\mathrm{OH}$ stretch vibration increases while the peak becomes broader and lower wavenumber. $\mathrm{OH}$ groups both in PLLA chain and in Glu results in various hydrogen bonding interactions and broadens $\mathrm{OH}$ peak of the blend.

The crystallinity of PLLA can be assessed by comparing the peak area at $756 \mathrm{~cm}^{-1}$ due to the crystalline phase with the peak area at $871 \mathrm{~cm}^{-1}$ due to amorphous phase. The peak area ratio between crystalline phase and amorphous phase increases steadily with increasing Glu. The bands at 956 and $922 \mathrm{~cm}^{-1}$ in PLLA spectrum have been separately ascribed to the amorphous and crystalline phase. With increasing Glu, the band at $956 \mathrm{~cm}^{-1}$ becomes very weak in blend spectra, while the band at $922 \mathrm{~cm}^{-1}$ enhances and shifts to lower wavenumber, which means that the crystallization of PLLA-Glu blends is enhanced ${ }^{[9]}$. IR results show that several kinds of hydrogen bonds between $\mathrm{C}=\mathrm{O}$ group of PLLA and $\mathrm{OH}$ group in both PLLA and Glu, which indicates that PLLA and Glu are partially compatible. Moreover, with the increase of glucose, the crystallization of the blends increased.

\section{B. Thermal Stability of PLLA, Glucose and Their Blends}

Fig. 2a and Fig. 2b showed thermo-gravimetric curve (TG) and DTG curve respectively, which were carried out to investigate the thermal degradation of PLLA-Glu blend. DTG profile confirms single step degradation process of PLLA blends. Thermo-gravimetric parameters were evaluated from the thermograms. The range of temperatures for the thermal degradation was estimated from the DTG curves, while the weight loss was determined from the TG plots. The $\mathrm{TG}$ and DTG curves of PLLA show $\mathrm{T}_{-5 \%}$ of $335^{\circ} \mathrm{C}, \mathrm{T}_{\max }$ of $370^{\circ} \mathrm{C}$ and $\mathrm{R}_{600}$ of $0.6 \%$. The TG and DTG curves of glucose show $\mathrm{T}_{-5 \%}$ of $217, \mathrm{~T}_{\max }$ of $317^{\circ} \mathrm{C}$ and $\mathrm{R}_{600}$ of $13.48 \%$, and a weak peak at $218^{\circ} \mathrm{C}$. With the addition of Glu, the $\mathrm{T}_{-5 \%}$ of PLLA-Glu blend is greatly reduced, which means the thermal stability of PLLA-Glu blend decreases with the addition of Glu. On the other hand, PLLA and PLLA-Glu blends possess similar $\mathrm{T}_{\max }$, which denotes that PLLA may play more important role at high decomposition temperature. There are two thermal decomposition peaks in DTG curve of PLLA-Glu blends, which may be caused by the decomposition of glucose at lower temperature ${ }^{[2]}$. The weight residue of PLLA-Glu blend increases gradually with increasing Glu. TG and DTG results show that the addition of glucose promotes thermal degradation of PLLA due to the trans-esterification reaction between glucose containing hydroxyl groups and PLLA at high temperature, which can reduce PLLA molecular weight and promote the thermal degradation of PLLA. Moreover, the addition of glucose can destroy PLLA crystalline structure to obtain imperfect PLLA crystalline, which also reduces the stability of PLLA 
[9]. All these results indicate that partial compatibility between glucose and PLLA performs great effect on the structure and properties of PLLA-Glu blends.

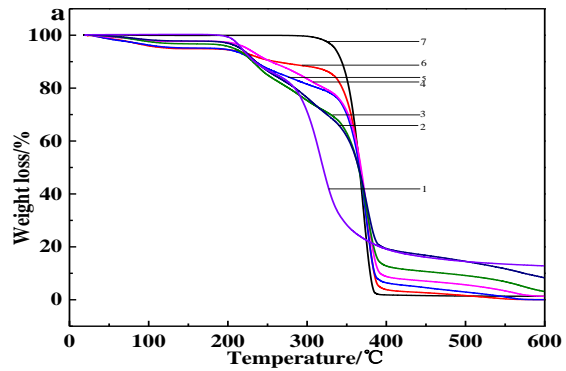

a. TG curve

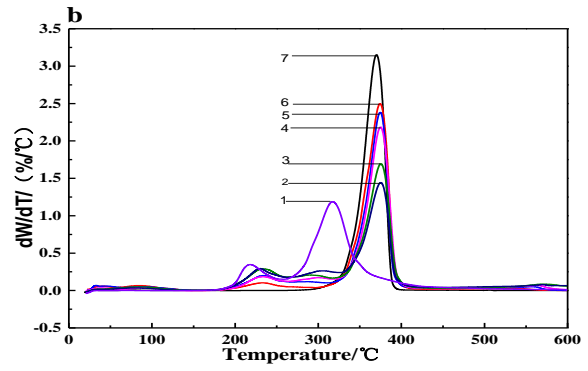

b. DTG curve.

Figure 2. Thermo-gravimetric curves of PLLA, Glu and blends Mass ratios of PLLA/Glu: 1. Glu, 2. 5:5, 3. 8:2, 4. 6:4, 5. 7:3, 6. 9:1, 7. PLLA

\section{DSC Analysis of PLLA, Glucose and PLLA-Glu Blends}

Fig. 3 gives DSC curves of PLLA, glucose and PLLA-Glu blends. The DSC curve of PLLA displays a glass transition temperature $\left(T_{\mathrm{g}}\right)$ at $60^{\circ} \mathrm{C}$, a melting point $\left(T_{\mathrm{m}}\right)$ at $154^{\circ} \mathrm{C}$ due to its crystal structure ${ }^{[10]}$. The curve of glucose displays a melting point at $165^{\circ} \mathrm{C}$ without $T_{\mathrm{g}}$. All DSC curves of PLLA-Glu blends show a single $T_{\mathrm{g}}$ and melting point. With increasing glucose, $T_{\mathrm{g}}$ of the blends increases firstly to the maximum as glucose content being $30 \%$, and then decreases with the increase of glucose. $T_{\mathrm{m}}$ of the blends, lower than that of both Glu and PLLA, decreases firstly and then increases with glucose content. These results show that glucose and PLLA have considerable interaction, namely PLLA and glucose are partly compatible. A small quantity of glucose acting as impurity may destroy the crystallization of PLLA, which results in imperfect crystal of PLLA. On the other hand, a large quantity of glucose acting as the nucleator during PLLA crystallization can improve the crystallization of PLLA. There are two melting peaks in the curves of the blends as glucose content more than $30 \%$, while the high temperature peak due to the recrystallization of PLLA to form more stable crysta ${ }^{[11]}$ caused by glucose. The result of DSC shows that there is a strong interaction between PLLA and glucose, which is consistent with the analysis of IR spectra.

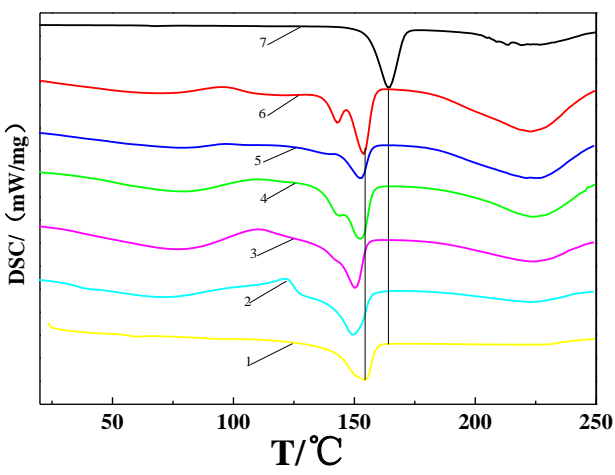

Figure 3. DSC curves of PLLA, Glu and PLLA-Glu blends Mass ratios of PLLA/Glu: 1. PLLA, 2. 9:1, 3. 8:2, 4. 7:3, 5. 6:4, 6. 5:5, 7. Glucose

\section{X-Ray Diffraction Analysis of PLLA, Glucose and} PLLA-Glu Blends

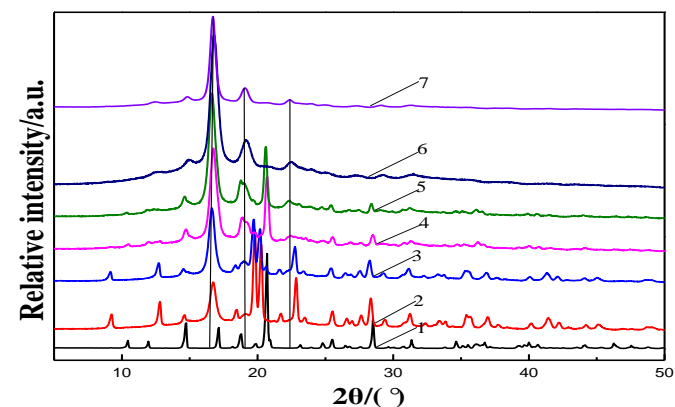

Figure 4. XRD curves of PLLA, glucose and PLLA-Glu blends Mass ratios of PLLA/Glu: 1. Glu, 2. 5:5, 3. 6:4, 4. 7:3, 5. 8:2, 6. 9:1, 7. PLLA

Fig. 4 gives XRD curves of PLLA, glucose and PLLA-Glu blends. The XRD curve of glucose has sharp diffraction peaks with the strongest at $2 \theta$ of $20.5^{\circ}$ for glucose possesses high crystallinity 98.16\%. PLLA is $\alpha$-crystalline structure with the strongest peaks at $2 \theta$ of $16.52^{\circ}$, as well as other peaks at $14.90^{\circ}, 19.21^{\circ}$ and $22.45^{\circ}$ with a crystallinity of $20.8 \%$. The XRD curve of PLLA-Glu blend with PLLA/Glu ratio of 9:1 is similar to that of PLLA ${ }^{[9,12]}$. This result is probably owing to the strong interaction between PLLA and glucose, which may hinder the crystallization process of glucose and cause imperfect crystal of PLLA, as already demonstrated by DSC. When glucose content increases, XRD curves of PLLA-Glu blends show peaks due to PLLA crystal and glucose crystal, which means that glucose crystal can appear due to excessive glucose as well as PLLA crystal. Moreover, both the peak at $2 \theta$ of $16.52^{\circ}$ and the peak at $19.21^{\circ}$ of PLLA shift to lower angle, which may indicate that the strong interaction between glucose and PLLA in the blends may cause imperfect crystal of PLLA and glucose. XRD results are completely consistent with those of FT-IR and DSC.

\section{CONCLUSION}

PLLA-Glu blends were prepared by mixed solution method, and the structure, crystallization, thermal 
properties, morphology and compatibility of PLLA-Glu blends were investigated. The results show that there is strong interaction such as hydrogen-bonding between PLLA and glucose, while the two components of PLLA-Glu blend are partially compatible. The crystallization of the blends increased with the increase of glucose. The addition of glucose can promote thermal degradation of PLLA due to phase defect of PLLA caused by glucose impurity or trans-esterification between PLLA and glucose.

\section{ACKNOWLEDGEMENT}

This paper is funded by Natural Science Foundation of China (50573032, 31200451); Forestry industry research special funds projects for public welfare (201204803), A Project Funded by the Priority Academic Program Development of Jiangsu Higher Education Institutions.

\section{REFERENCES}

[1] R. G. F. Costa, G. S. Brichi, C. Ribeiro, L. H. C. Mattoso, "Nanocomposite fibers of poly(lactic acid)/titanium dioxide prepared by solution blow spinning”, Polymer Bulletin, vol. 73, Nov. 2016, pp. 2973- 2985.

[2] P. M. Ma, T. F. Shen, L. Lin, W. F. Dong, M. Q. Chen, "Cellulose-gpoly(D-lactide) nanohybrids induced significant low melt viscosity and fast crystallization of fully bio-based nanocomposites", Carbohydrate Polymers, vol. 155, Jan. 2017, pp. 498-506

[3] W. D. Ding, D. Jahani, E. Chang, A. Alemdar, C. B. Park, M. Sain, "Development of PLA/cellulosic fiber composite foams using injection molding: Crystallization and foaming behaviors", Composites, Part A vol. 83, Apr. 2016, pp. 130-139

[4] P. Chaiwutthinan, V. Pimpan, S. Chuayjuljit, and T. Leejarkpai, "Biodegradable Plastics Prepared from Poly(lactic acid),
Poly(butylene succinate) and Microcrystalline Cellulose Extracted from Waste- Cotton Fabric with a Chain Extender", Journal of Polymers \& the Environment, vol. 23, Mar. 2015, pp. 114-125.

[5] Q. W. Gao, M. Z. Li, X. Dong, and J, Qian, "Study on biodegradable material from Poly(L-lactic acid)- ethyl cellulose blends", Chemistry and industry of forest products, vol. 25, Dec. 2005, pp. 17-20.

[6] H. Ebadi-Dehaghani, M. Barikani, S. Borhani, B. Bolvardi, H. A. Khonakdar, S. H. Jafari, A. Aarabi, "Biodegradation and hydrolysis studies on polypropylene/ polylactide/ organo-clay nanocomposites", Polymer Bulletin, vol. 73, Dec. 2016, pp. 3287-3304

[7] I. Spiridon, O. M. Paduraru, M. F. Zaltariov, and and R. N. Darie, "Influence of Keratin on Polylactic Acid /Chitosan Composite Properties. Behavior upon Accelerated Weathering", Industrial \& Engineering Chemistry Research, vol. 52, Jun. 2013, pp. 9822-9833.

[8] F. R. Beltran, V. Lorenzo, M. U. de la Orden, J. Martinez-Urreaga, "Effect of different mechanical recycling processes on the hydrolytic degradation of poly(L-lactic acid)", Polymer Degradation and Stability, vol.133, Nov. 2016, pp. 339-348.

[9] Q. Li, R. Zhang, C. Shao, Y.M. Wang, and C.Y. Shen. "Cold crystallization behavior of glassy poly(lactic acid) prepared by rapid compression", Polymer Engineering \& Science, vol. 55, Feb. 2015, pp. 359-366.

[10] R. Valapa, G. Pugazhenthi, and V. Katiyar, "Thermal degradation kinetics of sucrose palmitate reinforced poly(lactic acid) biocomposites", International Journal of Biological Macromolecules, vol. 65, Apr. 2014, pp. 275-283.

[11] R. Mat Taib, Z. A. Ghaleb, Z. A. Mohd Ishak, "Thermal, mechanical, and morphological properties of polylactic acid toughened with an impact modifier", Journal of Applied Polymer Science, vol. 123, Mar, 2012, pp. 2715-2725.

[12] A. N. Frone, D. M. Panaitescu, I. Chiulan, C. A. Nicolae, Z. Vuluga, C. Vitelaru, C. M. Damian, "The effect of cellulose nanofibers on the crystallinity and nanostructure of poly(lactic acid) composites", Journal of Materials Science, Vol. 51, Nov. 2016, pp. 9771-9791. 